\title{
Simulating a Chaotic Process
}

\author{
Ricardo L. Viana, José R. R. Barbosa*, \\ Departamento de Física, Universidade Federal do Paraná \\ 81531-990, Curitiba, Paraná, Brazil \\ Celso Grebogi, \\ Instituto de Física, Universidade de São Paulo \\ 05315-970, São Paulo, São Paulo, Brazil \\ and Antônio M. Batista, \\ Departamento de Matemática e Estatística, Universidade Estadual de Ponta Grossa, \\ 84033-240, Ponta Grossa, Paraná, Brazil \\ Received on 7 April, 2004

\begin{abstract}
Computer simulations of partial differential equations of mathematical physics typically lead to some kind of high-dimensional dynamical system. When there is chaotic behavior we are faced with fundamental dynamical difficulties. We choose as a paradigm of such high-dimensional system a kicked double rotor. This system is investigated for parameter values at which it is strongly non-hyperbolic through a mechanism called unstable dimension variability, through which there are periodic orbits embedded in a chaotic attractor with different numbers of unstable directions. Our numerical investigation is primarily based on the analysis of the finite-time Lyapunov exponents, which gives us useful hints about the onset and evolution of unstable dimension variability for the double rotor map, as a system parameter (the forcing amplitude) is varied.
\end{abstract}

\section{Introduction}

Computer simulations of partial differential equations are a main tool in the hands of physicists, playing an increasingly important role in their formation [1]. There are plenty of physical problems where computer simulations have an outstanding position, like in the study of turbulence in fluids and plasmas [2], percolation [3], statistical mechanics [4], and molecular dynamics [5], just to cite a few. Many numerical methods for numerical solution of partial differential equations rely on some form of discretization of space and/or time, such that those methods boil down to a system of coupled ordinary differential equations or coupled maps, in the case of a continuous or discrete time variable, respectively [6].

Nonlinear dynamical systems, including many partial differential equations of physical interest, commonly present chaotic motion, i.e. extreme sensitivity to initial conditions [7]. Since there are unavoidable errors inherent to the process of discretizing a partial differential equation, besides the usual one-step integration errors, the computer simulation of such equations may lead to fundamental dynamical difficulties. One of the aims of the present paper is to present the basic ideas and results underlying the study of the dynamical problems related to high-dimensional sys- tems undergoing chaotic motion.

We will choose as a paradigm of a high-dimensional dynamical system a mechanical system consisting of a double rotor subjected to periodic impulsive excitation, in which we adress the most severe of those dynamical difficulties, the so-called unstable dimension variability [8]. The approach we choose to investigate the onset, evolution and effects of unstable dimension variability in dynamical system is to consider the behavior of their numerically computed finite-time Lyapunov exponents.

Finite-time, or time- $n$, Lyapunov exponents are obtained exactly in the same way as the usual exponents, but over a finite time interval instead of the infinite-time limit always supposed in calculations [9]. Obviously, in practice any computed exponent is a finite-time one, but here we are concentrating in very small time intervals, in the $n=1$ to 100 range. The usefulness of finite-time exponents result from their interpretation as local expansion or contraction rates of a given trajectory during a small interval of time $[10,11]$. Hence, it is particularly sensitive to dynamical situations wherein the phase space presents a complicated structure of invariant sets with different numbers of stable and unstable directions.

This is the case, for example, of strongly non-hyperbolic dynamical systems presenting unstable dimension variabil-

* Permanent address: Departamento de Matemática, Universidade Federal do Paraná, 81531-990, Curitiba, Paraná, Brazil 
ity, which is characterized by the simultaneous occurrence, within the same invariant chaotic set, of unstable periodic orbits with a different number of unstable eigendirections [12]. It turns out that, as a typical chaotic trajectory wanders through the invariant set, it experiences alternate contractions and expansions with respect to the eigendirection which varies from point to point in the set. This is reflected in a fluctuating behaviour (around zero) of the corresponding finite-time Lyapunov exponent, since it is the local expansion rate for that eigendirection.

We remark that there are severe consequences of unstable dimension variability on the validity of numerically generated trajectories (which have small yet unavoidable one-step errors), since they fail to be shadowed by fiducial chaotic trajectories for a sufficiently large timespan [13]. In cases where a physical system is found to present unstable dimension variability we are thus able to closely follow a computer-generated trajectory for a time so short that it cannot be taken individually as a faithful representation of the actual dynamical behavior we are investigating [14]. Even statistical averages computed with help of non-shadowable chaotic trajectories may be doubtful since tiny errors may be largely amplified by system dynamics [15].

Unstable dimension variability was first described in a dynamical system of physical interest, a periodically kicked double rotor [16], and later on it was recognized that a fingerprint of unstable dimension variability, when it occurs at all, is the fluctuating behavior (around zero) of the finitetime Lyapunov exponent closest to zero [17]. It must be stressed, however, that it may happen that such exponents may fluctuate in such way without being a clearcut characterization of unstable dimension variability, since this fluctuations can come from other sources of non-hyperbolic behavior [18]. Hence, the onset and evolution of unstable dimension variability should be also sought for in the stability analysis of unstable periodic orbits embedded in the invariant chaotic set, and which support the ergodic measure of the orbits whose initial conditions lie in that set.

Nevertheless, the analysis of finite-time Lyapunov exponents has been proved to be very useful to give numerical insights on the onset and evolution of unstable dimension variability, especially in high-dimensional dynamical systems (like coupled oscillator and map lattices), where unstable dimension variability has been found to be very common [19]. The purpose of this paper is to investigate the onset and evolution of unstable dimension variability in the kicked double rotor map, as one system parameter (the forcing amplitude) is varied. We will base our analysis on the behavior of the finite-time Lyapunov exponents, as well as on the stability analysis, whenever it is possible due to the highdimensionality of the system, of the relevant low-period unstable orbits. Two basic issues are highlighted through this work: the onset of unstable dimension variability, which we conjecture to coincide with the onset of chaotic behavior itself; and the point where unstable dimension variability is most intense, namely, when occurs a codimension-one bifurcation within the chaotic set.

The rest of this paper is organized as follows: Section II presents the kicked double rotor system and the correspond- ing map equations, as well as some aspects of its chaotic dynamics. Section III discusses the onset and evolution of unstable dimension variability, when a system parameter is varied. The last section contains our conclusions.

\section{Kicked double rotor map}

The kicked double rotor system consists of two rods of negligible mass and of lengths $\ell_{1}$ and $\ell_{2}$, respectively (Fig. 1). The former rod, which has a point mass $m_{1}$ attached to one of its ends, is pivoted at the point $P_{1}$, and its angular position $\theta_{1} \in[0,2 \pi)$ is measured with respect to a reference line. The latter rod, with length $2 \ell_{2}$, is pivoted at its midpoint $P_{2}$ and has two point masses $m_{2} / 2$ attached to its free ends. The corresponding angular position, $\theta_{2}$, is measured with respect to a line parallel to the first reference line. We do not consider any gravity effects on this system (for example, it can move on a horizontal plane without friction).

An impulsive force

$$
f(t)=f_{0} \sum_{k=1}^{\infty} \delta(t-k T),
$$

with amplitude $f_{0} \geq 0$ and period $T$, is applied at one of the free ends, in a direction parallel to the reference line (cf. Fig. 1). Dissipation in this system occurs at the pivoting points $P_{1}$ and $P_{2}$ by means of viscous frictional forces. The friction at $P_{1}$, with damping coefficient $\nu_{1}>0$, slows down the first rod at a rate proportional to its angular velocity $\dot{\theta}_{1}$. The friction at the other pivot, $P_{2}$, slows down the second rod (but accelerates the first one) according to the relative angular velocities $\dot{\theta}_{2}-\dot{\theta}_{1}$, with damping coefficient $\nu_{2}>0$.

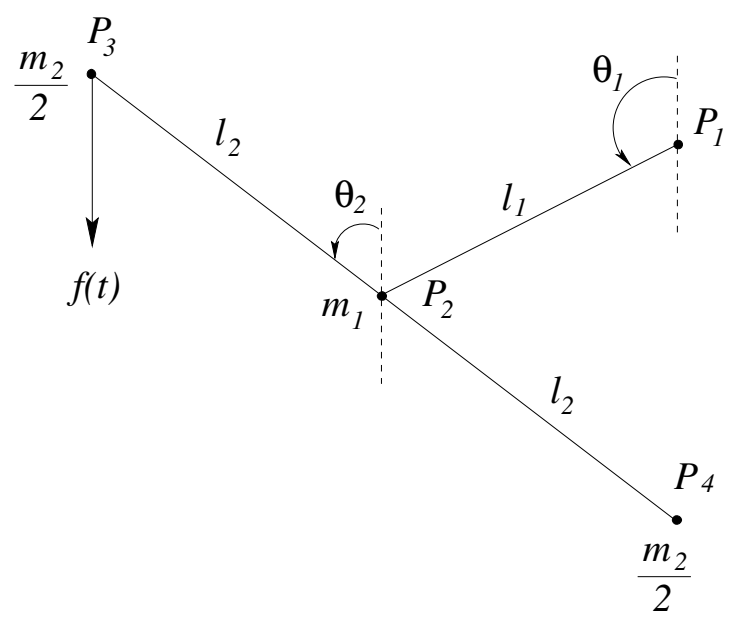

Figure 1. Sketch of a kicked double rotor.

We introduce the following discretized dynamical variables:

$$
\mathbf{X}_{n}=\left(\begin{array}{c}
\left(x_{1}\right)_{n} \\
\left(x_{2}\right)_{n}
\end{array}\right)=\lim _{\varepsilon \rightarrow 0}\left(\begin{array}{c}
\theta_{1}(n T+\varepsilon) \\
\theta_{2}(n T+\varepsilon)
\end{array}\right)
$$

representing the angular positions of the rotor rods just after the $n$-th impulsive kick, i.e., at discrete times $t=n T$ 
$(n=0,1,2, \cdots), T$ being the period of the delta-function excitation; and

$$
\mathbf{Y}_{n}=\left(\begin{array}{c}
\left(y_{1}\right)_{n} \\
\left(y_{2}\right)_{n}
\end{array}\right)=\lim _{\varepsilon \rightarrow 0}\left(\begin{array}{c}
\dot{\theta}_{1}(n T+\varepsilon) \\
\dot{\theta}_{2}(n T+\varepsilon)
\end{array}\right)
$$

being the corresponding discretized angular velocities.

Given the angular positions and velocities at time $n$, one can obtain the corresponding variables at the next instant $n+1$ by using the following map (further details on the obtention of this map can be found in Ref. [16]):

$$
\begin{aligned}
& \mathbf{X}_{n+1}=\mathbf{M} \mathbf{Y}_{n}+\mathbf{X}_{n} \\
& \mathbf{Y}_{n+1}=\mathbf{L} \mathbf{Y}_{n}+\mathbf{G}\left(\mathbf{X}_{n+1}\right)
\end{aligned}
$$

where the nonlinear functions are

$$
\mathbf{G}(\mathbf{X})=\left(\begin{array}{c}
\left(f_{0} \ell_{1} / I\right) \sin x_{1} \\
\left(f_{0} \ell_{2} / I\right) \sin x_{2}
\end{array}\right)
$$

and $I=\left(m_{1}+m_{2}\right) \ell_{1}^{2}=m_{2} \ell_{2}^{2}$ is the moment of inertia of the rotor.

The $2 \times 2$ constant matrices $\mathbf{L}$ and $\mathbf{M}$ are such that

$$
\mathbf{L}=\mathbf{I}_{2}+\mathbf{A}_{\nu} \mathbf{M}
$$

where $\mathbf{I}_{2}$ is the identity matrix, and

$$
\mathbf{A}_{\nu}=\left(\begin{array}{cc}
-\left(\nu_{1}+\nu_{2}\right) & \nu_{2} \\
\nu_{2} & -\nu_{2}
\end{array}\right)
$$

with

$$
\mathbf{M}=\sum_{j=1}^{2} \mathbf{W}_{j} \frac{\exp \left(s_{j} T\right)-1}{s_{j}}
$$

where

$$
s_{1,2}=-\frac{1}{2}\left(\nu_{1}+2 \nu_{2} \pm \Delta\right)
$$

are the eigenvalues of $\mathbf{A}_{\nu}$, with $\Delta \equiv \sqrt{\nu_{1}^{2}+4 \nu_{2}^{2}}$, and we have defined the following matrices

$$
\mathbf{W}_{1}=\left(\begin{array}{cc}
a & b \\
b & d
\end{array}\right), \quad \mathbf{W}_{2}=\left(\begin{array}{cc}
d & -b \\
-b & a
\end{array}\right)
$$

with constant entries

$$
a \equiv \frac{1}{2}\left(1+\frac{\nu_{1}}{\Delta}\right), \quad d \equiv \frac{1}{2}\left(1-\frac{\nu_{1}}{\Delta}\right), \quad b \equiv-\frac{\nu_{2}}{\Delta} .
$$

In the following, we simplify matters, without loss of generality, by taking convenient numerical values for the physical magnitudes involved:

$$
\ell_{1}=1 / \sqrt{2}, \quad \nu_{1}=\nu_{2}=T=I=m_{1}=m_{2}=\ell_{2}=1,
$$

such that

$$
\mathbf{L}=\left(\begin{array}{cc}
0.2414 & 0.2726 \\
0.2726 & 0.514
\end{array}\right), \quad \mathbf{M}=\left(\begin{array}{ll}
0.4860 & 0.2133 \\
0.2133 & 0.6993
\end{array}\right)
$$

As already mentioned, the control parameter to be varied is the driving amplitude $f_{0}$.
The dynamics of the kicked double rotor map (KDRM) is structured on their fixed points and periodic orbits. Due to the $y_{i} \rightarrow-y_{i}$ symmetry of the kicked double rotor map it turns out that the plane $y_{1}=y_{2}=0$ is an invariant subspace of the system, embedded in its four-dimensional phase space. Any initial conditions belonging to this plane will generate orbits which cannot escape from it as time evolves. Hence, at least some of the fixed points of the map, given by

$$
\begin{aligned}
& \mathbf{X}^{*}=\mathbf{M} \mathbf{Y}^{*}+\mathbf{X}^{*}(\bmod 2 \pi) \\
& \mathbf{Y}^{*}=\mathbf{L} \mathbf{Y}^{*}+\mathbf{G}\left(\mathbf{X}^{*}\right)
\end{aligned}
$$

must lie on this invariant subspace. The solutions of the above equations can be classified in one-parameter families [16]

$$
\mathbf{X}^{*}=\left(\begin{array}{c}
x_{1 *}^{\left(n_{1}, n_{2}, q\right)} \\
x_{2 *}^{\left(n_{1}, n_{2}, q\right)}
\end{array}\right) \quad \mathbf{Y}^{*}=\left(\begin{array}{c}
y_{1 *}^{\left(n_{1}, n_{2}\right)} \\
y_{2 *}^{\left(n_{1}, n_{2}\right)}
\end{array}\right)
$$

where $\left(n_{1}, n_{2}\right)$ are integer rotation numbers, and $q=$ $1,2,3,4$. In fact, four of these fixed points $\left(\mathbf{X}^{*}, \mathbf{Y}^{*}\right)$ lie on the $y_{1}=y_{2}=0$ plane. We will analyze particularly the fixed point with $n_{1}=n_{2}=0$ and $p=4$, called $\mathcal{P}=\left(x_{1}^{*}=\pi, x_{2}^{*}=\pi, y_{1}^{*}=0, y_{2}^{*}=0\right)$.

A representative part of the bifurcation diagram for the kicked double rotor map is depicted in Fig. 2, where one of the dynamical variables, namely the angular position of the second rod, $x_{2}$, is plotted versus the kick strength $f_{0}$ in the interval $[4.0,7.0]$. For small values of $f_{0}$ we observe the stable fixed point, $\mathcal{P}$, which undergoes a period doubling bifurcation at $f_{0} \approx 4.27$ producing a stable period- 2 orbit. The latter is followed, for higher forcing amplitudes, by a period-doubling cascade leading to chaos for $f_{0} \approx 6.75$. The chaotic bands merge together and eventually fuse themselves into a single large chaotic band, at $f_{0} \approx 6.85$ due to a crisis triggered by the collision of the two-band chaotic attractor with some unstable orbit.

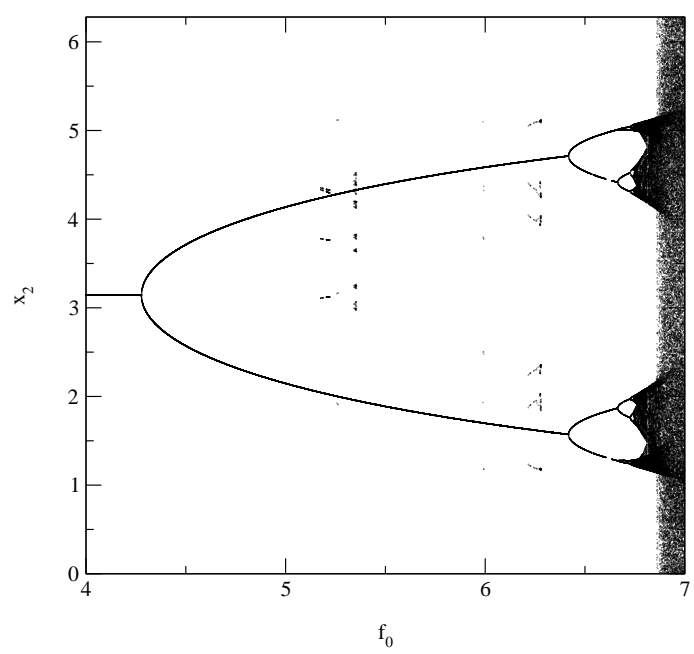

Figure 2. Bifurcation diagram of $x_{2}$ versus the control parameter $f_{0} \in[4.0,7.0]$.

Besides this main structure, we observe in Fig. 2, just after $f_{0}=5.2$, a coexisting periodic orbit born through 
a saddle-node bifurcation (i.e., there is also a companion unstable periodic orbit). This orbit undergoes a perioddoubling cascade (not shown in the figure due to insufficient graphical resolution) leading to a chaotic motion suddenly interrupted at $f_{0} \approx 5.4$ due to a crisis (caused by the collision between the chaotic attractor and the unstable orbit created at the saddle-node bifurcation). Another coexisting periodic orbit appears and disappears in the narrow interval $[6.2,6.3]$.

\section{Unstable dimension variability in the kicked double rotor map}

To introduce the key question to be discussed in this paper, let us make a simple numerical experiment with the kicked double rotor map: we choose a given value of the control parameter and an initial condition for which the map is known to generate a chaotic orbit. Next we iterate the map for a given number of times, using both single and double precision (see Fig. 3). It is seen that, after no more than 18 map iterates, the resulting orbits are far apart by a distance comparable to the length scale used of that phase space region. Since the only difference between these trajectories $\left(\sim 10^{-8}\right)$ has been the truncation furnished by the computer arithmetics we have chosen, one could understandably regard both orbits as equally suspicious, because a third and different trajectory would result from using another (e.g., quadruple) numerical precision. As this problem is not likely to end with an ever increased arithmetical precision, one could ask whether or not should we trust those computer-generated orbits?

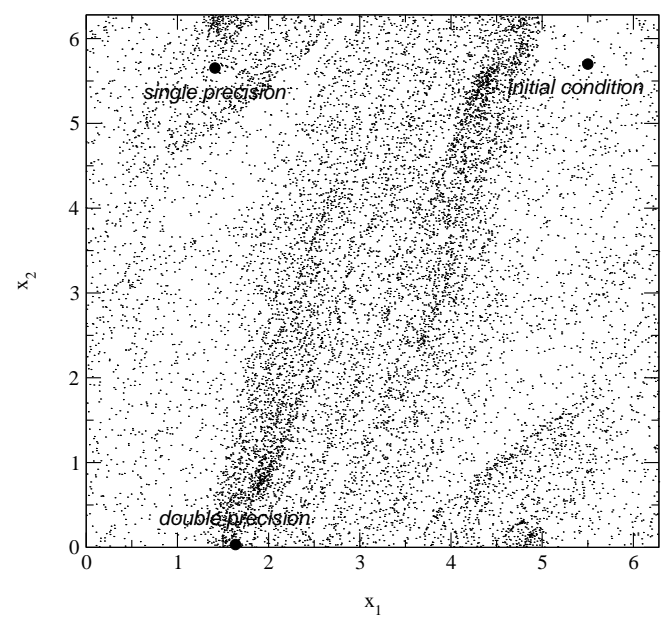

Figure 3. Projection, on the $\left(x_{1}, x_{2}\right)$ plane, of the phase points of two chaotic trajectories arising from the same indicated initial condition $(5.5,5.7,0.0,0.0)$ and iterated 18 times using single and double precision, for the $\operatorname{KDRM}\left(f_{0}=7.5\right)$.

This is obviously nothing but a consequence of the chaotic nature of the orbits. Consider that, for the value of control parameter used in plotting Fig. 3, the maximal Lyapunov exponent of the KDRM is $\lambda_{1} \approx 1.1$ [16]. The phase-space distance between the single and double precision trajectories after one iterate, which is of the order $10^{-8}$, grows exponentially as the time $n$ increases at a rate equal to the maximal Lyapunov exponent; in that way, the number of iterations for which the distance increases to a number of order 1 is

$$
\frac{1}{\lambda_{1}} \ln \left(\frac{1}{10^{-8}}\right) \sim 17
$$

in accordance with the result illustrated by Fig. 3 .

In spite of this, it is far from trivial to get the answer to the question about whether or not those computer-generated chaotic orbits are meaningful. In fact, the efforts to come to a comprehensive understanding of such problems have stimulated a branch of pure mathematics called "shadowing theory" [13]. There has been proved that only hyperbolic systems are shadowed by an arbitrarily large (in fact, infinite) timespan [20, 21]. When the dynamics fail to be hyperbolic due to glitches caused by near-tangencies between stable and unstable manifolds of an unstable orbit, it can still be argued that shadowing is possible during a timespan of the order $\delta^{-1 / 2}$, where $\delta$ is the magnitude of the one-step errors commited during numerical computation of that orbit [22]. If the system is non-hyperbolic due to unstable dimension variability, however, the shadowing time depends on the statistical properties of the finite-time Lyapunov exponents, and may be so short that no useful prediction can come from single chaotic trajectories [15].

In order to investigate the onset and evolution of unstable dimension variability in the kicked double rotor map, we will focus on the first representative chaotic attractor of the double rotor, which appears at $f_{0} \approx 6.75$ [see Fig. 2], where the maximal Lyapunov exponent $\lambda_{1}$ crosses zero and becomes positive [16]. Exception being made to periodic windows, such as those occurring at $7.0 \lesssim f_{0} \lesssim 7.2$ and around 8.2 , the exponent $\lambda_{1}$ is almost always positive indicating a reasonably steady chaotic dynamics for the system. As the forcing amplitude is further increased, it turns out that the second Lyapunov exponent, $\lambda_{2}$, builds up monotonically and crosses zero at $f_{0}^{*}=8.1104126$. This is recognized as the onset of hyper-chaos in the system, since there is more than one positive exponent. The unstable fixed point $\mathcal{P}$ is embedded in this chaotic attractor.

Computing the eigenvalue $\xi_{2}$ (the closest to unity in absolute value), of the linearized map for the fixed point $\mathcal{P}$, there results that, when $f_{0} \lesssim f_{0}^{*}=8.1104126, \mathcal{P}$ possesses three stable and one unstable direction, hence $d_{s}(\mathcal{P})=3$ and $d_{u}(\mathcal{P})=1$. Evaluating the eigenvalue $\xi_{2}$ in the neighborhood of the hyper-chaotic transition point $f_{0}^{*}$ (Fig. 4), there occurs a period-doubling bifurcation. For $f_{0} \gtrsim f_{0}^{*}$ the fixed point $\mathcal{P}$ acquires two stable and two unstable directions, i.e., its unstable dimension becomes $d_{u}(\mathcal{P})=2$. Moreover, there is an infinite number of pre-images of $\mathcal{P}$, embedded in the chaotic attractor of the system, but this set 
does not contain all points belonging to the attractor. In addition to this set of periodic orbits with $d_{u}=2$, there remains an infinite number of other periodic orbits that continue to have unstable dimension $d_{u}=1$. Since these sets are densely intertwined in the attractor, a typical chaotic trajectory will approach points with one or two unstable directions.

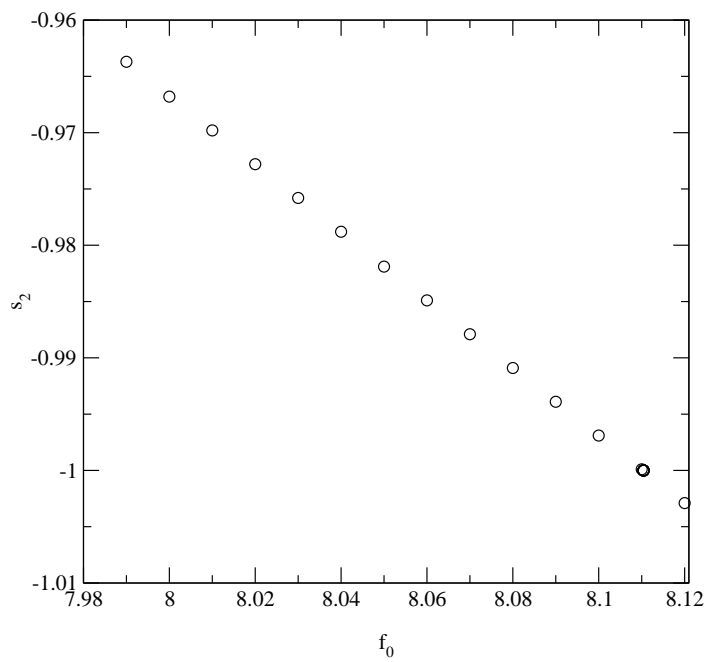

Figure 4. Eigenvalue of the Jacobian matrix of the KDRM as a function of the control parameter $f_{0}$ near the emergence of hyperchaos.

This is a direct evidence that unstable dimension variability occurs for the chaotic attractor of the double rotor when $f_{0} \gtrsim f_{0}^{*}$. However, as we will see in the next section, the onset of unstable dimension variability occurs before $f_{0}^{*}$. This is because there may occur variations on the unstable dimension of other fixed points or periodic orbits embedded in the chaotic attractor, before this happens for this selected fixed point $\mathcal{P}$. The problems with shadowability of numerical trajectories, in this case, follow from this complicated mixture of high-dimensional saddles and repellers. Consider a hyper-sphere in the four-dimensional phase space filled with initial conditions, whose evolution according to the double rotor map, generates "true", or fiducial chaotic trajectories. When this sphere approaches a given saddle $\left(d_{u}=1\right)$, it will shrink along three stable directions and elongate along one unstable direction, becoming a cigar-like tube centered at the saddle (Fig. 5) [23].

A numerically generated, or pseudo-trajectory $A$, will be contained inside this cigar-like tube of radius $\delta$ ( $\delta$ being the one-step error level commited during numerical iteration of the map), and this tube will also contain a true orbit B which will shadow $A$, since their pointwise distance is bounded by $\delta$. After a further number of iterations, the cigar-like tube will approach a repeller $\left(d_{u}=2\right)$ and will rapidly expand along the extra unstable direction. In this case, the pointwise distance between $A$ and $B$ will be no longer bounded and increases exponentially along the new unstable dimension. As a consequence, $A$ will be no longer shadowed by $B$, for they diverge exponentially with time. Since the sets of saddles and repellers are dense in the chaotic attractor, the time during which we preserve shadowing may be extremely short.
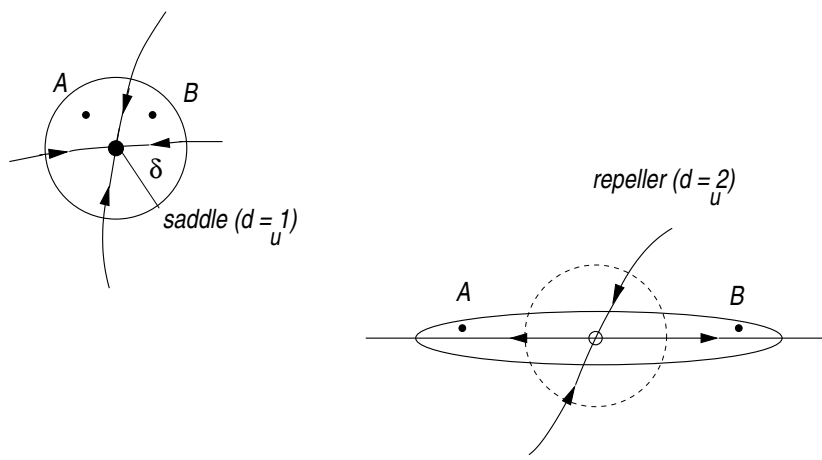

Figure 5. Schematic illustration of the evolution of a cigar-like tube of trajectories (which cross-section in the page plane is a circle) in presence of unstable dimension variability. The direction perpendicular to the page is repulsive.

These observations about expansion and contraction of phase space volumes can be made more quantitative by introducing the corresponding finite time Lyapunov exponents. Let $\mathbf{f}(\mathbf{x})$ be a $d$-dimensional map, where $n$ is a positive integer, such that $\mathbf{D f}^{n}\left(\mathbf{x}_{0}\right)$ is the Jacobian matrix of the $n$-times iterated map, with entries evaluated at the initial condition $\mathbf{x}_{0}$. Suppose that the singular values of $\mathbf{D} \mathbf{f}^{n}\left(\mathbf{x}_{0}\right)$ are ordered: $\xi_{1}\left(\mathbf{x}_{0}, n\right) \geq \xi_{2}\left(\mathbf{x}_{0}, n\right) \ldots \geq \xi_{n}\left(\mathbf{x}_{0}, n\right)$. Then, the $k$-th time- $n$ Lyapunov exponent for the point $\mathbf{x}_{0}$ is defined as [24]

$$
\lambda_{k}\left(\mathbf{x}_{0}, n\right)=\frac{1}{n} \ln \left\|\mathbf{D} \mathbf{f}^{n}\left(\mathbf{x}_{0}\right) \cdot \mathbf{v}_{k}\right\|,
$$

where $\mathbf{v}_{k}$ is the eigenvector corresponding to $\xi_{k}\left(\mathbf{x}_{0}, n\right)$.

The infinite time-limit of the above expression is the usual Lyapunov exponent

$$
\lambda_{k}(\infty)=\lim _{n \rightarrow \infty} \lambda_{k}\left(\mathbf{x}_{0}, n\right)
$$

Although the time- $n$ exponent $\lambda_{k}\left(\mathbf{x}_{0}, n\right)$ generally takes on a different value, depending on the point we choose, the infinite time limit takes on the same value for almost all $\mathbf{x}_{0}$ with respect to the natural ergodic measure of the invariant set. For the kicked double rotor map there are four time- $n$ exponents, ordered as $\lambda_{1}(n)>\lambda_{2}(n)>\lambda_{3}(n)>\lambda_{4}(n)$, but we will be interested only in the closest to zero, which in this case turns out to be $\lambda_{2}(n)$.

A numerical indication of unstable dimension variability is the fluctuating behavior (around zero) of the time- $n$ Lyapunov exponent closest to zero [17]. This follows from the fact that there are unstable points with different unstable dimensions, such that a typical trajectory on the chaotic (or hyper-chaotic) attractor will visit neighborhoods of radius $\epsilon$ of saddles and repellers in the attractor for any $\epsilon$, no matter how small. This means that there are time- $n$ segments for which the trajectory is transversely attracting (on 
average) and others for which it is transversely repelling (also on average). This is properly quantified by the time- $n$ Lyapunov exponent closest to zero $\lambda_{2}(n)$ : when the trajectory sections of duration $n$ are such that there is an average contraction (expansion) along its eigendirection, the corresponding time- $n$ exponent is negative (positive). Hence, if the chaotic invariant set displays unstable dimension variability, there will be length- $n$ sections of a typical trajectory for which $\lambda_{2}(n)$ is positive, even when the infinite-time exponent $\lambda_{2}(\infty)$ is negative. This suggests the use of a probability distribution $P\left(\lambda_{2}(n)\right)$, such that $P\left(\lambda_{2}(n)\right) d \lambda_{2}(n)$ is the probability that the second time- $n$ exponent lies between $\lambda_{2}(n)$ and $\lambda_{2}(n)+d \lambda_{2}(n)$.

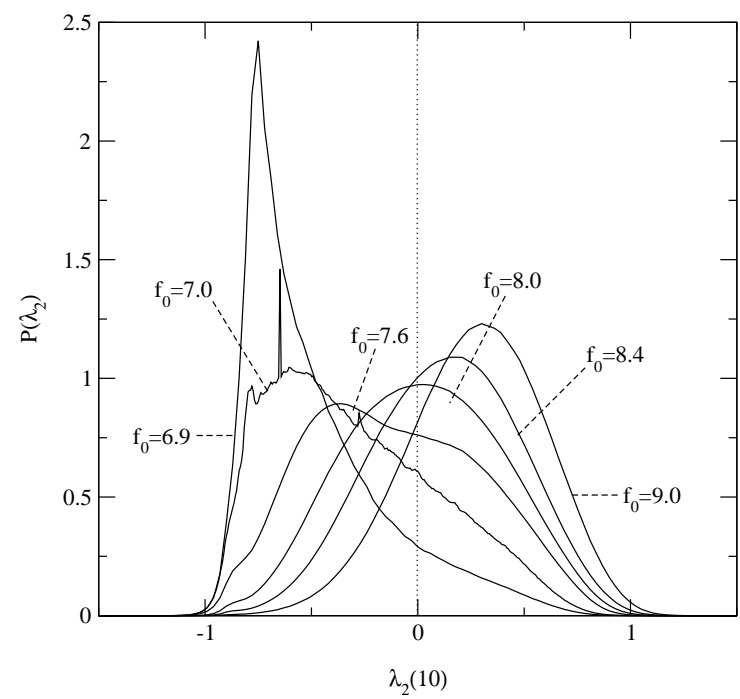

Figure 6. Probability distribution for the second finite-time Lyapunov exponent, $\lambda_{2}(10)$, for the kicked double rotor map.

We can obtain a numerical approximation for this probability distribution, for the double rotor map, by means of a histogram drawn on a large number of trajectories of length $n$ from randomly chosen initial conditions. In Fig. 6, we show a few distributions of time-10 exponents, obtained for different values of the forcing amplitude $f_{0}$, chosen in a parameter interval roughly centered at the onset of hyper-chaos $\left(f_{0}^{*}\right)$, the bell-shape of $P\left(\lambda_{2}(n)\right)$ being more evident as we approach this point. The maxima of the distributions drift towards positive values, as $f_{0}$ varies in the vicinity of the hyper-chaos transition $f_{0}^{*}$. Fig. 7 plots the average values of the distributions of the time-10 exponents along the four eigendirections, $\left\langle\lambda_{i}(n)>, i=1,2,3,4\right.$. This figure is very similar to the corresponding diagram for the infinitetime Lyapunov exponents [16, 23]. In fact, for Gaussian distributions it follows that [8]

$$
\left\langle\lambda_{2}\left(\mathbf{x}_{0}, n\right)\right\rangle=\frac{\int_{-\infty}^{+\infty} \lambda_{2}\left(\mathbf{x}_{0}, n\right) P\left(\lambda_{2}\left(\mathbf{x}_{0}, n\right)\right) d \lambda_{2}}{\int_{-\infty}^{+\infty} P\left(\lambda_{2}\left(\mathbf{x}_{0}, n\right)\right) d \lambda_{2}}=\lambda_{2}(\infty)
$$

Figure 7 points out that the average time- $n$ exponent crosses zero at a constant rate in the neighborhood of the hyper-chaos transition. Since the distributions depicted in Fig. 6 have most of the $\lambda_{2}(n)$-values in the $[-1,+1]$ range, there results a considerable distortion of these distributions, with respect to a Gaussian shape. This is confirmed by Fig. 8, which plots the variance $\sigma_{n}^{2}$ of the $P\left(\lambda_{2}(n)\right)$ distributions in the same range, showing a highly fluctuating behavior before hyper-chaos, and a mostly smooth decrease before it. In other dynamical systems for which unstable dimension variability has been studied $[25,26]$, the distributions of the corresponding time- $n$ exponent of interest, besides being Gaussian-shaped, drift towards positive values without noticeable distortion of shape.

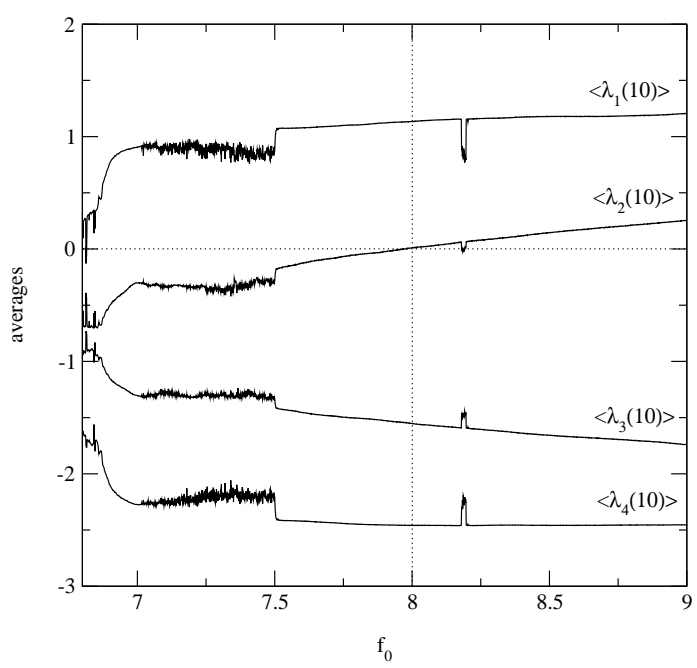

Figure 7. Average time-10 Lyapunov exponents for the kicked double rotor map, as a function of the forcing amplitude. $\lambda_{2}(n)$

A quantity of interest is the fraction of positive values of

$$
\phi(n)=\frac{\int_{0}^{\infty} P\left(\lambda_{2}(n)\right) d \lambda_{2}(n)}{\int_{-\infty}^{+\infty} P\left(\lambda_{2}\left(\mathbf{x}_{0}, n\right)\right) d \lambda_{2}}
$$

We can obtain an approximate location for the onset of unstable dimension variability, as a system parameter is continuously varied, as the parameter value for which the fraction $\phi(n)$ becomes nonzero. Fig. 9 depicts a numerical approximation of this fraction for the double rotor map, indicating is apparently at a value $f_{0}=\left(f_{0}\right)_{C} \approx 6.8$. The second (infinite-time) Lyapunov exponent in the interval between 6.8 and 8.0 is negative [16], indicating that unstable dimension variability actually starts before the onset of hyper-chaos. 


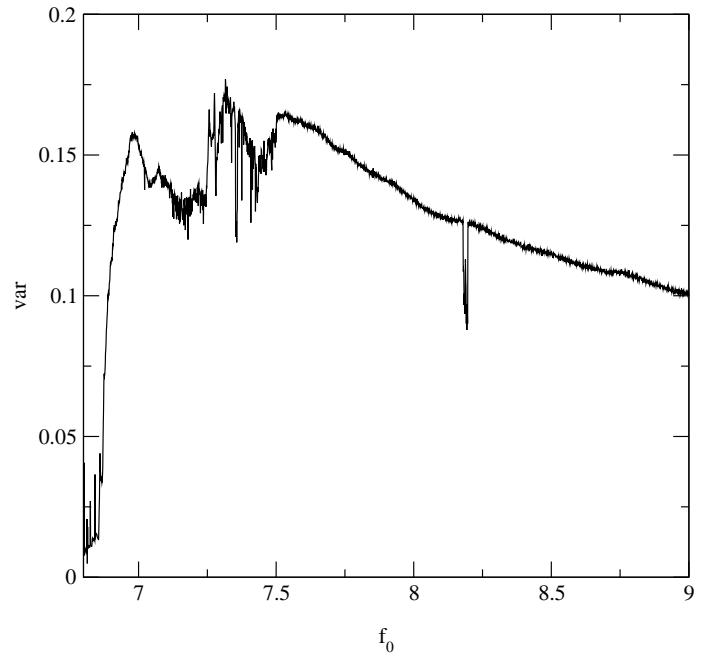

Figure 8. Variance of the second time-10 Lyapunov exponents for the kicked double rotor map, as a function of the forcing amplitude.

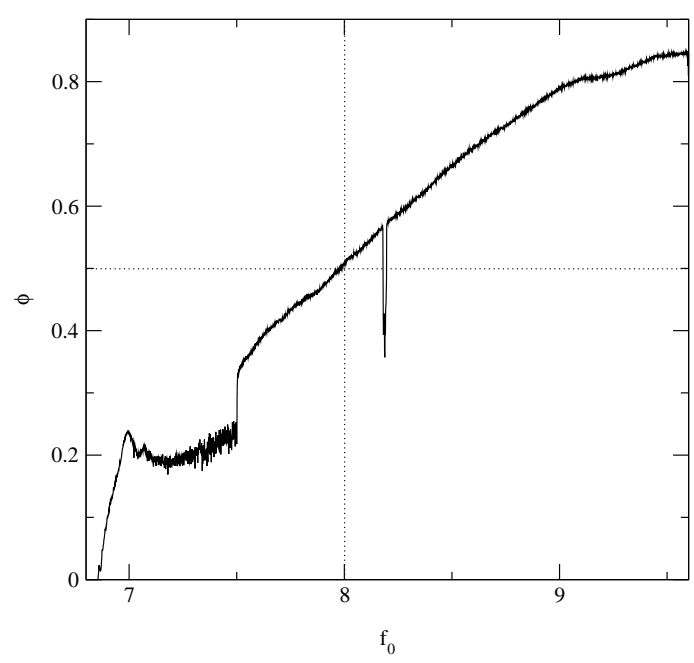

Figure 9. Fraction of positive second time-10 Lyapunov exponents for the kicked double rotor map, as a function of the forcing amplitude.

The transition to hyper-chaos is identified in Fig. 9 with a positive fraction of $50 \%$ of the exponents. In fact, Fig. 6 shows, for this value, a distribution with symmetric tails whose maximum crosses the $\lambda_{2}(n)=0$ line. When half of the time- $n$ exponents are negative, given a certain value of $\lambda_{2}(n)$ in modulus, say 0.2 , there is an approximately equal probability of this value be positive or negative. In other words, the relative weight of local expansions or contractions is roughly the same, what is the worst situation when one tries to obtain a "true" chaotic trajectory which shadows a numerical one. This argument can be made more precise by assigning these relative contributions of contractions and expansions the weights of unstable periodic orbits with different unstable dimensions by computing the natural measure of the chaotic attractor $[23,8]$.

Hence, the onset of hyper-chaos does not coincide, in general, with the onset of UDV, but the former is rather the point at which the effect of UDV is of maximum strength. This point marks also the blowout bifurcation, at which the chaotic set as a whole loses transversal stability (the eigendirection related to $\lambda_{2}$, in the double rotor map) [27]. The parameter interval between the onset of unstable dimension variability and its maximum value $\left(\left(f_{0}\right)_{C}<f_{0}<f_{0}^{*}\right.$ for the KDRM) is related to the bubbling phenomenon, when the chaotic set is contained in an invariant subspace of the system [28]. For $f_{0}>f_{0}^{*}$, according to Fig. 9, the fraction of positive exponents continues to increase, and so the contrast between negative and positive values. In this case, the effect of unstable dimension variability (for example, in terms of the shadowability properties of chaotic trajectories) becomes progressively less pronounced, even though the two higher infinite-time Lyapunov exponents increase in this region. Curiously, it appears that strongly hyper-chaotic trajectories are best shadowed than those at the verge of the hyper-chaos transition.

The above observations can be made more quantitative by using the concept of shadowing time, defined as the time interval $\tau$ during which a $\delta$-pseudo-trajectory is shadowed by a "true" one. Sauer and co-workers [14] have developed a theoretical model of the pseudo-trajectory behavior in the KDRM by considering a biased stochastic diffusion process with a reflecting barrier, the latter playing the role of the one-step errors of level $\delta$ which bound the noisy pseudotrajectory. They estimated an average shadowing time scaling as power law, $\left\langle\tau>\sim \delta^{-h}\right.$, where it has been defined the hyperbolicity exponent [15]

$$
h \equiv \frac{2<\lambda_{2}(n)>}{\sigma_{1}^{2}}
$$

in terms of the average and variance, respectively, of the distribution $P\left(\lambda_{2}(1)\right)$ of the time-1 Lyapunov exponent closest to zero. The latter is related to the variance of the time- $n$ exponent by $\sigma_{n}^{2}=n \sigma_{1}^{2}$.

In the vicinity of the blowout bifurcation point, where $\left.<\lambda_{2}(n)\right\rangle \approx 0$, the hyperbolicity exponent is nearly zero, and the shadowing time can be so small as a few iterations of the map, hence the effects unstable dimension variability are indeed more intense at these points. As we move apart from this point, the shadowing time increases in a nonuniform fashion due to the peculiar behavior of the average and variance (see Figs. 7 and 8, respectively). Nevertheless, the occurrence of unstable dimension variability yields values of $\tau$ which are usually too small to give us confidence on long-time averages made using computer generated trajectories. Even extremely small errors propagate at an exponential rate and plague any statistics made on the basis of such chaotic trajectories [15]. 

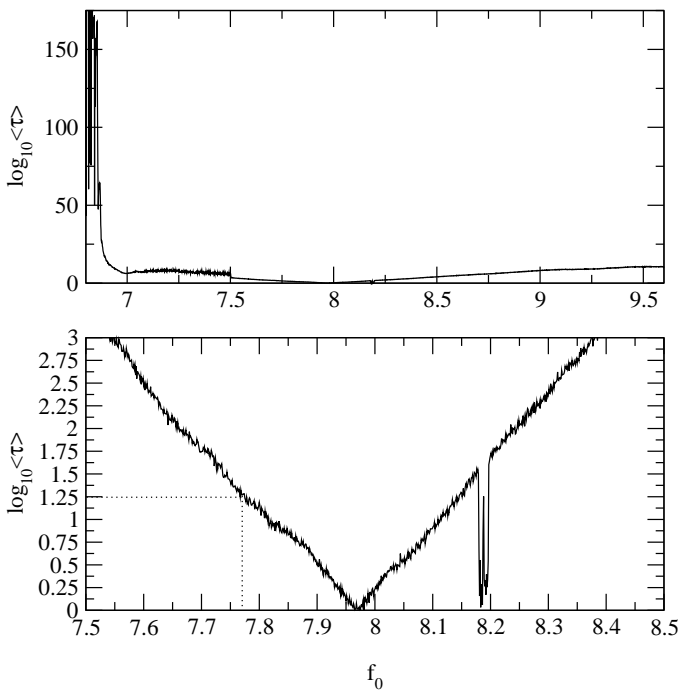

Figure 10. Average shadowing time for the kicked double rotor map, as a function of the forcing amplitude. Bottom panel: magnification of the interval for which unstable dimension variability is most intense.

\section{Conclusions}

We have discussed in the last Section the issue of finding "true" trajectories of a chaotic system which can shadow, for a time long enough, a numerically generated pseudotrajectory of the same model. However, we can also think of these two trajectories as coming from different models of a same physical phenomenon. If the computer generated trajectory is a $\delta$-pseudo trajectory, it can also be regarded as a "true" trajectory which stems from a slightly perturbed version of the original model. This perturbation, or modeling error, whose intensity is bounded by a number of the order of $\delta$, can come from different sources [29].

One possibility is that the trajectory to be shadowed comes from a perturbed version of the model, resulting from either: (i) a parameter imperfect determination; (ii) a small change in the external influence on the model. In the KDRM case, a perturbed version of the model can be due to (i) a small change in the one of the masses or lengths; whereas (ii) could stand for an experimental uncertainty related with the determination of the forcing amplitude, for example. The noisy trajectory is then due to a value of $f_{0}$ determined within an accuracy proportional to $\delta$. Another possibility (iii) is to regard the noisy trajectory as coming from a replica of the KDRM which has been corrupted by an external noise term bounded by $\delta$ (this excludes unbounded, e.g. Gaussian, noise).

In both cases, the same concepts of shadowability applied to pairs of trajectories can be extended to pairs of models. One mathematical model $\mathcal{A}$ of a physical phenomenon (like the KDRM) is then said to shadow the mathematical model $\mathcal{B}$, which turns to be a slightly perturbed version of $\mathcal{A}$, if the set of all possible outcomes (or trajectories coming from typical initial conditions) from model $\mathcal{A}$ agrees closely with the set of all possible outcomes from model $\mathcal{A}$. In terms of the $\epsilon$-shadowing property of trajectories, this means that for every trajectory of model $\mathcal{A}$ there exists at least one trajectory of model $\mathcal{B}$ that $\epsilon$-shadows the particular trajectory of model $\mathcal{A}$, and vice-versa.

As a consequence, UDV represents a serious obstacle also for model shadowability, since there are trajectories from one model, as $\mathcal{A}$, that do not $\epsilon$-shadow any trajectory from the other one $(\mathcal{B})$ for all but extremely short periods of time. Considering that trajectories from both models do not agree with each other in terms of shadowability, it is unlikely that either $\mathcal{A}$ or $\mathcal{B}$ is a good model for the physical phenomenon it intends to describe, like the kicked double rotor, for no model trajectory shadows any true trajectory of the actual physical system being investigated.

We stress that this uselessness of mathematical models is not due to the inadequacy of the physical hypotheses used in formulating the model, nor the incorrect application (for example, out of the proper range) of the physical laws, not even due to neglecting subtle but nevertheless key factors underlying a good modeling. Unstable dimension variability is a mathematical pathology that hampers model shadowability, and which is rather unavoidable, as long as the mechanical system produces chaotic trajectories.

Hence, even though such a model was intended to describe a deterministic system, it will produce at best the same kind of statistical information a stochastic system would yield, as averages and fluctuations. We can call mathematical models of chaotic systems displaying unstable dimension variability as pseudo-deterministic systems, of which the kicked double rotor is a representative example. Such systems may not display model shadowability in a satisfactory degree for sets of parameter values, and should be avoided when we are making predictions about the future state of the system based on individual trajectories. In these cases, one could resort to time-series analysis methods to reconstruct the attractor from experimental data, like phase space embedding using delay coordinates.

\section{Acknowledgments}

This work was made possible through partial financial support from the following Brazilian research agencies: FAPESP and CNPq. C. G. also acknowledges support from Humboldt Foundation. The authors are indebted to S. R. Lopes, S. E. de S. Pinto and J. Kurths for useful discussions and suggestions.

\section{References}

[1] S. Koonin and D. Meredith, Computational Physics, Perseus Books, 2002.

[2] U. Frisch, Turbulence : The Legacy of A. N. Kolmogorov, Cambridge University Press, Cambridge, 2001. 
[3] D. Stauffer and A. Aharony, Introduction to Percolation Theory, Taylor and Francis, London, 1994.

[4] S. Moss de Oliveira, P.M.C. de Oliveira and D. Stauffer, Evolution, Money, War and Computers, Teubner, LeipzigStuttgart, 1999.

[5] D. C. Rapaport, The Art of Molecular Dynamics Simulation, Cambridge University Press, Cambridge, 2001.

[6] W. H. Press, B. P. Flanger, S. A. Teulkosky, and W. T. Vetterling, Numerical Recipes: The art of scientific computing. Cambridge University Press, Cambridge, 1986.

[7] L. Lam, Nonlinear Physics for Beginners, World Scientific, Singapore, 1998.

[8] R. L. Viana, C. Grebogi, S. E. S. Pinto, and J. R. R. Barbosa, Int. J. Bifurcat. Chaos 13, 3235-3253 (2003).

[9] A. Wolf, J. B. Swift, H. L. Swinney, and J. A. Vastano, Physica 16D, 285-317 (1985).

[10] H. Fujisaka, Prog. Theor. Phys. 70, 1264 (1983).

[11] A. Prasad and R. Ramaswany, Phys. Rev. E 60, 2761 (1999).

[12] R. Abraham and S. Smale, Proc. Symp. Pure Math. (AMS) 14, 5 (1970).

[13] C. Grebogi, L. Poon, T. Sauer, J. A. Yorke, and D. Auerbach, in Handbook of Dynamical Systems, Ed. B. Fiedler (Elsevier, Amsterdam, 2002) Vol. 2, Chapt. 7.

[14] T. Sauer, C. Grebogi, and J. A. Yorke, Phys. Rev. Lett. 79, 59 (1997).

[15] T. Sauer, Phys. Rev. E 65, 036220 (2002).

[16] F. J. Romeiras, C. Grebogi, E. Ott, and W. P. Dayawansa, Physica D 58, 165 (1992)
[17] S. P. Dawson, C. Grebogi, T. Sauer, and J. A. Yorke, Phys. Rev. Lett. 73, 1927 (1994).

[18] H. D. I. Abarbanel, R. Brown, and M. B. Kennel, J. Nonlinear Sci. 1, 175 (1991).

[19] Y.-C. Lai, D. Lerner, K. Williams, and C. Grebogi, Phys. Rev. E 60, 5445 (1999).

[20] D. V. Anosov, Proc. Steklov Inst. Math. 90,1 (1967).

[21] R. Bowen, J. Diff. Eqs. 18, 333 (1975).

[22] C. Grebogi, S. Hammel, and J. A. Yorke, J. Complexity 3, 136 (1987); Bull. Am. Math. Soc. 19, 465 (1988). C. Grebogi, S. Hammel, J. A. Yorke, and T. Sauer, Phys. Rev. Lett. 65, 1527 (1990). T. Sauer and J. A. Yorke, Nonlinearity 4, 961 (1991).

[23] Y.-C. Lai and C. Grebogi, Int. J. Bifurcat. Chaos 10, 683 (2000); Y.-C. Lai, C. Grebogi, and J. Kurths, Phys. Rev. E 59, 2907 (1999).

[24] E. J. Kostelich, I. Kan, C. Grebogi, E. Ott, and J. A. Yorke, Physica D 109, 81 (1997).

[25] R. L. Viana and C. Grebogi, Phys. Rev. E 62, 462 (2000).

[26] R. L. Viana and C. Grebogi, Int. J. of Bifurcat. Chaos 11, 2689 (2001).

[27] P. Ashwin, J. Buescu, and I. Stewart, Phys. Lett. A 193, 126 (1994).

[28] S. E. S. Pinto, R. L. Viana, and C. Grebogi, Phys. Rev. E 66, 046213 (2002).

[29] L. Poon, S. P. Dawson, C. Grebogi, T. Sauer, and J. A. Yorke, in Dynamical Systems and Chaos, Eds. Y. Aizawa, S. Saito, K. Shiraiwa (World Scientific, Singapore, 1994). 